\title{
A possible tertiary rRNA interaction between expansion segments ES3 and ES6 in eukaryotic 40S ribosomal subunits
}

\author{
GUNNAR ALKEMAR ${ }^{1,2}$ and ODD NYGÅRD ${ }^{1}$ \\ ${ }^{1}$ Cell Biology Unit, Natural Science Section, Södertörns högskola, S-141 89 Huddinge, Sweden \\ ${ }^{2}$ Department of Cell Biology, Arrhenius Laboratories E5, Stockholm University, S-106 91 Stockholm, Sweden
}

\begin{abstract}
Eukaryotic 16S-like ribosomal RNAs contain 12 so-called expansion segments, i.e., sequences not included in the RNA secondary structure core common to eubacteria, archaea, and eukarya. Two of these expansion segments, ES3 and ES6, are juxtaposed in the recent three-dimensional model of the eukaryotic $40 S$ ribosomal subunit. We have analyzed ES3 and ES6 sequences from more than 2900 discrete eukaryotic species, for possible sequence complementarity between the two expansion segments. The data show that ES3 and ES6 could interact by forming a helix consisting of seven to nine contiguous base pairs in almost all analyzed species. We, therefore, suggest that ES3 and ES6 form a direct RNA-RNA contact in the ribosome.
\end{abstract}

Eukaryotic $18 \mathrm{~S}$ ribosomal RNA is considerably longer than its prokaryotic homolog $16 \mathrm{~S}$ rRNA (Clark 1987). Despite differences in size and sequence, $18 \mathrm{~S}$ rRNA and 16S rRNA share a common structural core (Gutell et al. 1985) in which the additional nucleotides found in 18S rRNA are inserted at specific positions. The inserted nucleotides form extra sequence elements called variable regions (Neefs and De Wachter 1990) or expansion segments (ES; Gerbi 1996). The length and sequence of these expansion segments varies considerably between organisms.

18S rRNA contains 12 expansion segments (Gerbi 1996). One of these expansion segments, referred to as ES6, is located in the central domain of $18 \mathrm{~S}$ rRNA (Fig. 1). With an average length of 250 nucleotides (Neefs and De Wachter 1990), this is the largest expansion segment found in $18 \mathrm{~S}$ rRNA. ES6 can be divided in two halves based on sequence variability. The $5^{\prime}$ half exhibits extensive variability, whereas the sequence of the $3^{\prime}$ half is more conserved. Sequences corresponding to the latter half are absent in eubacterial and archaeal 16S rRNA (Cannone et al. 2002). Several attempts to construct a secondary structure model for the $3^{\prime}$ half have been made (Nickrent and Sargent 1991; Hancock and

Reprint requests to: Odd Nygård, Cell Biology unit, Natural Science Section, Södertörns högskola, Box 4101, S-141 04 Huddinge, Sweden; email: odd.nygard@sh.se; fax: 4686084510.

Article and publication are at http://www.rnajournal.org/cgi/doi/ 10.1261/rna.2108203.
Vogler 1998; Wuyts et al. 2000). However, because of the sequence conservation, structure prediction is difficult, and this part of ES6 is therefore left unstructured in the phylogenetic models of $18 \mathrm{~S}$ rRNA secondary structure (Fig. 1; Cannone et al. 2002).

The location of ES6 within the three-dimensional structure of the yeast $40 \mathrm{~S}$ subunit has recently been determined using cryo-electron microscopy (Spahn et al. 2001). The expansion segment is located on the back of the so-called body of the 40S subunit (Fig. 1, inset). The segment is seen as two separate densities, one positioned across the back of the $40 \mathrm{~S}$ body, whereas the second density is located at the side of the lower part of the body with its lower end close to the so-called left foot (Spahn et al. 2001).

The left foot contains another expansion segment, called ES3 (Spahn et al. 2001), only found in eukaryotes (Cannone et al. 2002). ES3 is very variable in size and sequence (Gutell et al. 1985; Wuyts et al. 2002), but the phylogenetic data indicate a similar basic structure for ES3 in different organisms (Fig. 1; Cannone et al. 2002). The functions of ES3 and ES6 are unknown at present.

In the recent three-dimensional structure of the $80 \mathrm{~S}$ ribosome from yeast (Spahn et al. 2001), ES3 is located close to one of the densities formed by ES6 (Fig. 1, insert). This location indicated that sequences in ES3 and ES6 might be in direct contact on the $40 \mathrm{~S}$ ribosomal subunit. A search for complementary sequences in ES3 and ES6 in 18S rRNA from wheat (Triticum aestivum) and mouse (Mus musculus) 

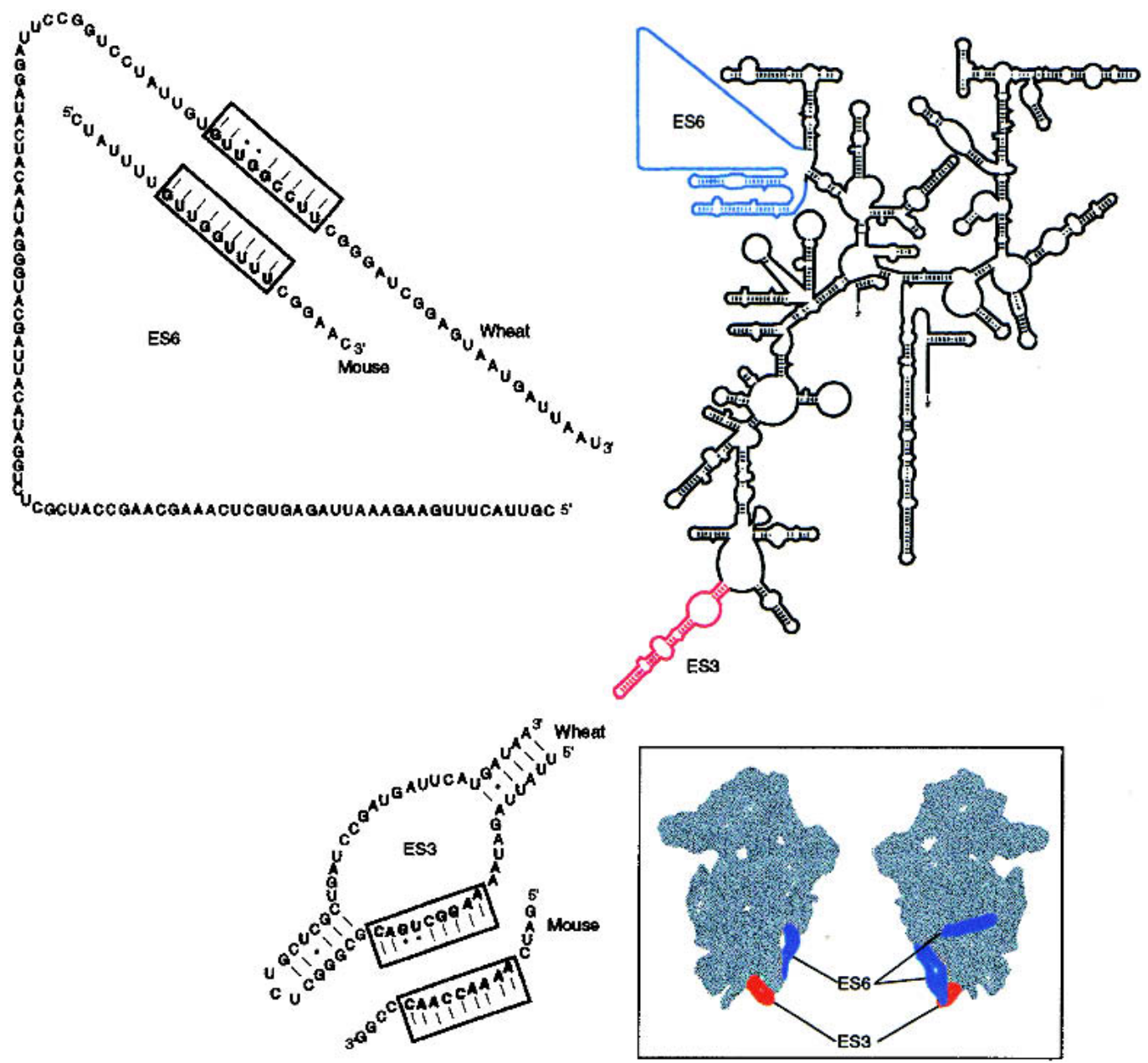

FIGURE 1. Secondary structure model of $18 \mathrm{~S}$ rRNA (Gutell 1993) showing the position of expansion sequences ES3 (red) and ES6 (blue) (Gerbi 1996). The sequences of the proposed complementary regions in ES3 and ES6 are shown for wheat Triticum aestivum and mouse Mus musculus, GenBank accession nos. AY049040 and X00686, respectively. (Inset) Position of ES3 (red) and ES6 (blue) in the 40S ribosomal subunit (Spahn et al. 2001). The $40 \mathrm{~S}$ subunit is shown from the inter-subunit side (left) and from the solvent side (right).

showed that matching sequences were found at homologous positions in ES3 and ES6 from both organisms (Fig. 1). In wheat, the sequence $\mathrm{A}^{216} \mathrm{AGGCUGAC}{ }^{224}$ in ES3 was complementary to nucleotides $\mathrm{G}^{839} \mathrm{UUGGCCUU}^{847}$ in ES6. The homologous sequences in mouse involved nucleotides $\mathrm{A}^{231}$ AAACCAAC $^{239}$ and $\mathrm{G}^{893} \mathrm{UUGGUUUU}^{901}$ (Fig. 1). Thus, the two expansion segments could form a 9-bp helix in both organisms, although the sequences of the complementary elements were not completely conserved.

The existence of similar base complementarities in ES3 and ES6 from other streptophytes than wheat was analyzed using the aligned 18S rRNA sequences available in the European Small Subunit Ribosomal Database (http:// rrna.uia.ac.be/ssu/index.html; Wuyts et al. 2002). As seen in Figure 2, the sequence of the two putative complementary regions in ES3 and ES6 was essentially conserved in all streptophytes. The sequence variation seen at positions 4,7 , and 8 in ES3, with adenine and guanine as the dominating bases (Fig. 2), did not affect the putative base-pairing ca- pacity of the ES3 sequence, as the base at the indicated complementary positions in ES6 was a conserved uracil. Similarly, the equal abundance of cytosine and uracil at position 9 in ES3 was compatible with a base pairing to the conserved guanine at position 1 in the ES6 sequence. However, in approximately half of the analyzed sequences, the ninth position in the ES6 element contained a guanine instead of a uracil (Fig. 2). A guanine was also the dominating base at the same position in chlorophytes. As the presumed interaction partner at position 1 in ES3 was a conserved adenine, the maximum number of possible base pairs between the two sequences was reduced to eight in most chlorophytes and in half of the streptophytes unless the putative helix is terminated by a non-Watson-Crick A - G base pair. Such base pairs are often found at helix termini in ribosomal RNA (Ban et al. 2000; Nagaswamy et al. 2000; Wimberly et al. 2000).

Even the two sequence elements found in mouse ES3 and ES6 were almost completely conserved in other chordates 


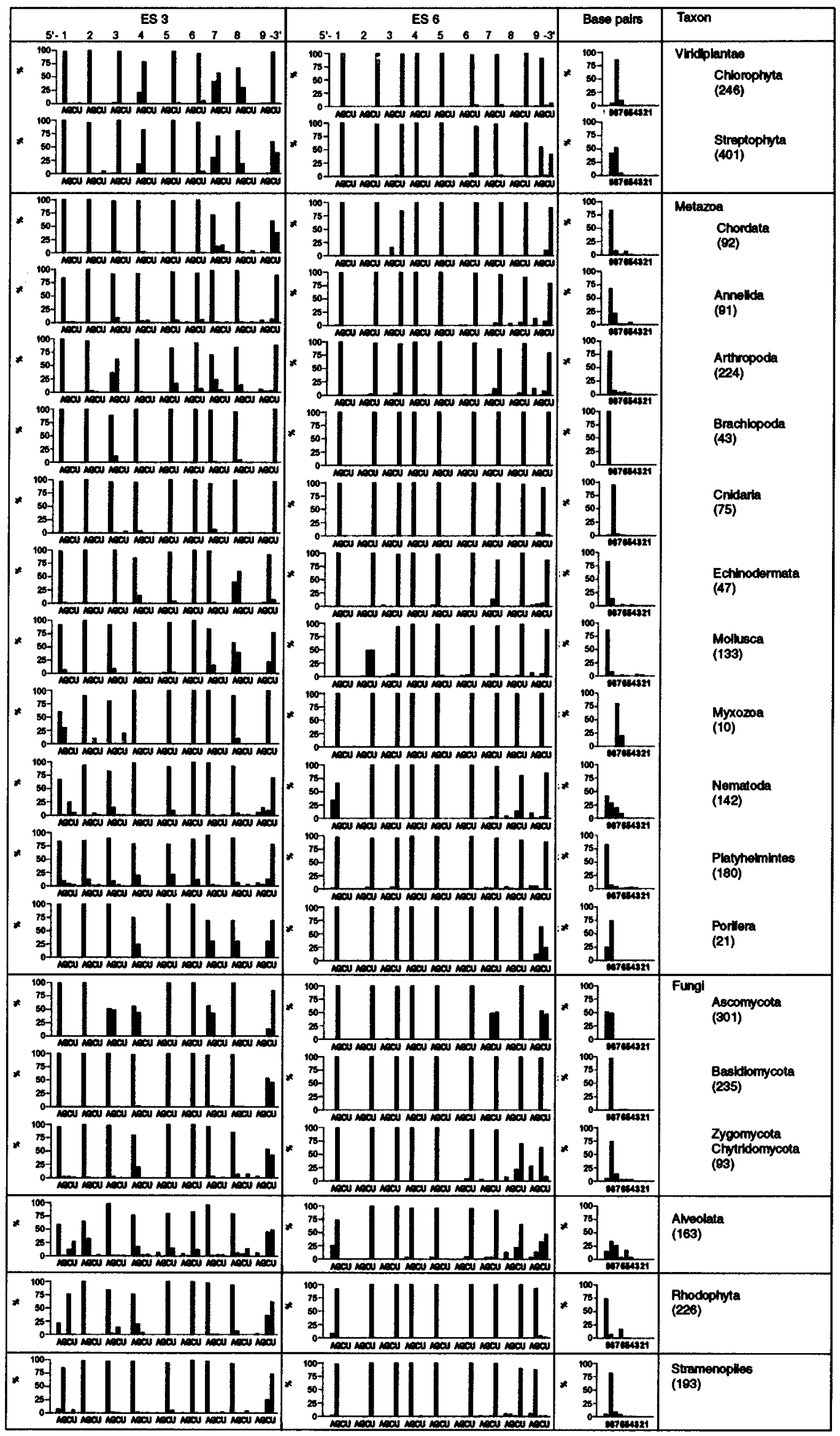

FIGURE 2. (legend on facing page) 
than mouse with the exception that cytosine and uracil were almost equally abundant at position 9 in the ES3 sequence (Fig. 2). As the putative interaction partner was a conserved guanine, this had no effect on the base-pairing capacity of the two sequences. Interestingly, some species had a cytosine at position 7 in ES3 instead of the normal purine (Fig. 2). This base change was compensated for by a change from uracil to a guanine in position 3 in the ES6 sequence.

An extended search for base complementarities at homologous sequence positions in 18S rRNA from other members of the large taxon Metazoa, showed that the two sequence elements in ES3 and ES6 were essentially conserved. Major differences from the consensus motifs (AAAACCAAU and GUUGGUUUU for ES3 and ES6, respectively) were seen in Cnidaria, Myxozoa, Nematoda, and Porifera. In Cnidaria and Porifera, the ninth base in the sequence from ES6 was frequently a cytosine instead of a uracil, whereas the base at the putative complementary position was always an adenine. This restricted the maximum length of the suggested interaction to $8 \mathrm{bp}$ in most of these organisms (Fig. 2). Nematodes and myxozoans showed the largest deviation from the consensus sequence. In nematodes the sequence of the ES3 element was relatively heterogeneous at positions 1 and 9, whereas the ES6 sequence remained unchanged. Thus, the maximum number of contiguous base pairs between the two sequence elements ranged from 7 to $9 \mathrm{bp}$ for most nematodes (Fig. 2). In myxozoans both the eighth and the ninth base in the ES6 element were cytosine rather than uracil. No compensatory base changes were seen at positions 1 and 2 in the ES3 element. Consequently, the maximum helical length was reduced to $7 \mathrm{bp}$. Compensatory base changes were, however, seen in molluscs. In these organisms, the base at position 2 in ES6 was often a cytosine instead of a uracil (Fig. 2). This base change was compensated for by a switch from adenine to guanine at position 8 in ES3 (Fig. 2).

The possible interaction between sequences in ES3 and ES6 from other taxa was also analyzed. Among the fungi, the consensus ES3 and ES6 sequences were AAAACCA $\mathrm{AC} / \mathrm{U}$ and GUUGGUUUC/U, respectively. In the basidiomycotes the occurrence of a cytosine at position 9 in the ES6 element restricted the putative number of base pairs to eight. A similar situation was also found among approximately half of the ascomycotes. The other heterogeneities observed in this group of organisms did not affect the potential base pairing. The situation was more complex in the group of Zygomycota/Chytridomycota. Here, the variability in base composition at positions 8 and 9 in the ES6 se- quence was not compensated for by a covariation at positions 1 and 2 in the ES3 sequence. Thus, the maximum length of the putative helix was reduced to $7-8$ consecutive base pairs.

As seen in Figure 2, both the ES3 and ES6 sequences in $18 \mathrm{~S}$ rRNA from the organisms in the taxon Alveolata showed extensive sequence heterogeneity. The heterogeneity was predominantly found at both ends of the two sequences. Despite this heterogeneity, the two sequences could form a 7-9-bp-long helix in most of these organisms even though there was a considerable portion of sequences that could only form a shorter stretch of contiguous base pairs. It should be noted that no species from the genus Plasmodium were included in this sequence comparison because it was difficult to identify the homologous ES3 sequence in the available alignment (Wuyts et al. 2002).

In rhodophytes the consensus sequence was CAAACCAAC/U and GUUGGUUUG for ES3 and ES6, respectively. Thus, in most of these organisms the purine found in position 1 in ES3 was changed to a pyrimidine. A compensatory change from a pyrimidine to a purine in position 9 in ES6 was also seen (Fig. 2). It was therefore still possible to form a 9-bp helix between ES3 and ES6 in most rhodophytes. A similar change from a pyrimidine to a guanine at position 9 in ES6 was also seen in stramenopiles (Fig. 2). However, in these organisms the putative complementary base in ES3 was also a guanine, thereby reducing the maximum helical length to 8 bp (Fig. 2).

High-resolution crystal structures of the prokaryotic ribosome (Ban et al. 2000; Wimberly et al. 2000) have revealed that the secondary structure models of $16 \mathrm{~S}$ rRNA (Gutell 1996) is largely correct. There are, however, far more tertiary interactions than originally predicted using comparative sequence analysis. The data presented here indicate that sequences in the two eukaryotic-specific expansion sequences ES3 and ES6 may form a tertiary interaction on the ribosome. The analyzed ES3 and ES6 sequences represent $>2900$ discrete species from six different eukaryotic taxa. The sequence variability for all investigated species is summarized in Table 1.

As seen in Table 1, the guanines at positions 4 and 5 in ES6 are conserved in almost all examined species. The putative complementary positions in ES3 always contain pyrimidines. Uracil is the dominating base at positions 2 and 3 and in positions 6-8 in ES6 with purines, most often adenine, at the complementary positions in ES3. In some species one or more of these uracils is replaced by cytosine. Almost all of these organisms have a guanine at the putative

FIGURE 2. Base composition and maximum length of the complementary sequences in ES3 and ES6 from different taxa. Sequence information for $>2900$ discrete species was taken from the European Small Subunit Ribosomal Database (http://rrna.uia.ac.be/ssu/index.html; Wuyts et al. 2002). (ES 3, ES 6) The relative abundance (\%) of each of the four bases at the nine positions in the two putative complementary sequences (ES3 and ES6). (Base pairs) The maximum possible number of continuous base pairs formed by the complementary sequences in ES3 and ES6. The bars represent the percentage of species within each group that could form a complementary helix of indicated length. The number of species in each taxon or group of organisms within a taxon is given in parentheses. The taxa listed correspond to the taxonomy usage given by the National Institute for Biotechnology Information (http://www.ncbi.nlm.nih.gov/Taxonomy/taxonomyhome.html). 
TABLE 1. Base composition of the two putative complementary sequences in ES3 and ES6.

\begin{tabular}{rrrrrrrrrrr}
\hline & \multicolumn{10}{c}{ Position } \\
\cline { 2 - 11 } & $\%$ & $5^{\prime}-1$ & 2 & 3 & 4 & 5 & 6 & 7 & 8 & $9-3^{\prime}$ \\
\hline \multirow{5}{*}{ ES3 } & $\mathrm{A}$ & 82 & 96 & 62 & 70 & & & 75 & 86 & 2 \\
& $\mathrm{G}$ & 7 & 4 & 37 & 29 & & & 24 & 11 & 1 \\
& $\mathrm{C}$ & 8 & & 1 & & 94 & 96 & & 1 & 42 \\
& $\mathrm{U}$ & 3 & & & & 5 & 3 & & 2 & 55 \\
& & & & & Position & & & & \\
\cline { 2 - 11 } & $\%$ & $3^{\prime}-9$ & 8 & 7 & 6 & 5 & 4 & 3 & 2 & $1-5^{\prime}$ \\
\hline \multirow{5}{*}{ ES6 } & $\mathrm{U}$ & 41 & 94 & 60 & 90 & & & 98 & 97 & \\
& $\mathrm{C}$ & 24 & 4 & 39 & 10 & & & 1 & 3 & \\
$\mathrm{G}$ & 31 & & & & 100 & 100 & & & 95 \\
& $\mathrm{~A}$ & 4 & 2 & & & & & & & 4 \\
\hline
\end{tabular}

The frequency (\%) is based on the examination of $>2900$ discrete species. Shaded areas represent the consensus sequence.

complementary position in ES3 (Table 1). Similarly, species in which the ES6 sequence has an adenine at position 1 also have a uracil in position 9 of ES3. The extensive variation seen at position 9 in ES6 was not compensated for by a similar variation at position 1 in ES3 (Table 1).

The sequence complementarity between ES3 and ES6 allows formation of a helix containing 7-9 contiguous base pairs in $>2700$ of the investigated organisms (Fig. 2). The indicated interaction between ES3 and ES6 is compatible with the most recently proposed secondary structure for ES6 (Wuyts et al. 2000). It is also compatible with the juxtaposition of ES3 and ES6 sequences seen in the threedimensional picture of the yeast $80 \mathrm{~S}$ ribosome (Spahn et al. 2001).

\section{ACKNOWLEDGMENTS}

The publication costs of this article were defrayed in part by payment of page charges. This article must therefore be hereby marked "advertisement" in accordance with 18 USC section 1734 solely to indicate this fact.

Received July 29, 2002; accepted September 30, 2002.

\section{REFERENCES}

Ban, N., Nissen, P., Hansen, J., Moore, P.B., and Steitz, T.A. 2000. The complete atomic structure of the large ribosomal subunit at $2.4 \AA$ resolution. Science 289: 905-920.

Cannone, J.J., Subramanian, S., Schnare, M.N., Collett, J.R., D’Souza, L.M., Du, Y., Feng, B., Lin, N., Madabusi, L.V., Muller, K.M., et al. 2002. The comparative RNA Web (CRW) site: An online database of comparative sequence and structure information for ribosomal, intron, and other RNAs. BMC Bioinformatics 3: 2.

Clark, C.G. 1987. On the evolution of ribosomal RNA. J. Mol. Evol. 25: $343-350$.

Gerbi, S.A. 1996. Expansion segments: Regions of variable size that interrupt the universal core secondary structure of ribosomal RNA. In Ribosomal RNA-Structure, evolution, processing, and function in protein synthesis (eds. R.A. Zimmermann and A.E. Dahlberg), pp. 71-87. CRC Press, Boca Raton, FL.

Gutell, R.R. 1993. Collection of small subunit (16S- and 16S-like) ribosomal RNA structures. Nucleic Acids Res. 21: 3051-3054.

- 1996. Comparative sequence analysis and the structure of $16 \mathrm{~S}$ and 23S rRNA. In Ribosomal RNA-Structure, evolution, processing, and function in protein synthesis (eds. R.A. Zimmermann and A.E. Dahlberg), pp. 111-128. CRC Press, Boca Raton, FL.

Gutell, R.R., Weiser, B., Woese, C.R., and Noller, H.F. 1985. Comparative anatomy of 16-S-like ribosomal RNA. Prog. Nucleic Acids Res. Mol. Biol. 32: 155-216.

Hancock, J.M. and Vogler, A.P. 1998. Modelling the secondary structures of slippage-prone hypervariable RNA regions: The example of the tiger beetle $18 \mathrm{~S}$ rRNA variable region V4. Nucleic Acids Res. 26: $1689-1699$.

Nagaswamy, U., Voss, N., Zhang, Z.D., and Fox, G.E. 2000. Database of non-canonical base pairs found in known RNA structures. Nucleic Acids Res. 28: 375-376.

Neefs, J.M. and De Wachter, R. 1990. A proposal for the secondary structure of a variable area of eukaryotic small ribosomal subunit RNA involving the existence of a pseudoknot. Nucleic Acids Res. 18: $5695-5704$.

Nickrent, D.L. and Sargent, M.L. 1991. An overview of the secondary structure of the V4 region of eukaryotic small-subunit ribosomal RNA. Nucleic Acids Res. 19: 227-235.

Spahn, C.M., Beckmann, R., Eswar, N., Penczek, P.A., Sali, A., Blobel, G., and Frank, J. 2001. Structure of the 80 S ribosome from Saccharomyces cerevisiae - tRNA-ribosome and subunit-subunit interactions. Cell 107: 373-386.

Wimberly, B.T., Brodersen, D.E., Clemons, W.M., Morgan-Warren, R.J., Carter, A.P., Vonrhein, C., Hartsch, T., and Ramakrishnan, V. 2000. Structure of the 30S ribosomal subunit. Nature 407: 327-339.

Wuyts, J., De Rijk, P., Van de Peer, Y., Pison, G., Rousseeuw, P., and De Wachter, R. 2000. Comparative analysis of more than 3000 sequences reveals the existence of two pseudoknots in area V4 of eukaryotic small subunit ribosomal RNA. Nucleic Acids Res. 28: 4698-4708.

Wuyts, J., Van de Peer, Y., Winkelmans, T., and De Wachter, R. 2002. The European database on small subunit ribosomal RNA. Nucleic Acids Res. 30: 183-185. 

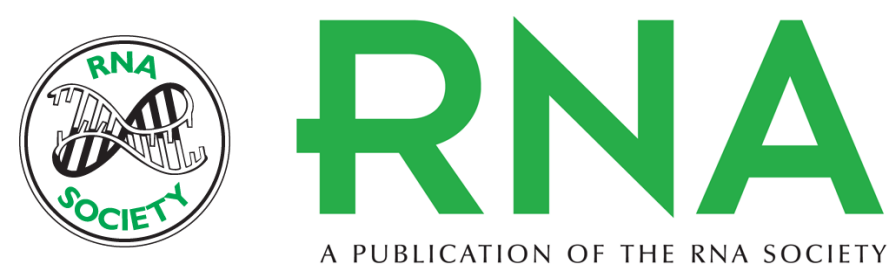

A PUBLICATION OF THE RNA SOCIETY

\section{A possible tertiary rRNA interaction between expansion segments ES3 and ES6 in eukaryotic 40S ribosomal subunits}

GUNNAR ALKEMAR and ODD NYGÅRD

RNA 2003 9: 20-24

$\begin{array}{ll}\text { References } & \begin{array}{l}\text { This article cites } 13 \text { articles, } 1 \text { of which can be accessed free at: } \\ \text { http://rnajournal.cshlp.org/content/9/1/20.full.html\#ref-list-1 }\end{array}\end{array}$

License

Email Alerting Receive free email alerts when new articles cite this article - sign up in the box at the Service top right corner of the article or click here. 